\title{
Partenariat entre professionnels et parents d'enfants avec une déficience intellectuelle
}

\author{
Expériences et attentes des parents
}

\section{Partnership between professionals and parents of mentally} retarded children

\section{Parents' experiences and needs}

\section{Cooperación entre los profesionistas y los padres de familia con niños que presentan una deficiencia intelectual Experiencias y esperanzas de los padres de familia}

\author{
Gisela Chatelanat, Isaline Panchaud Mingrone et Gertrude Niggl Domenjoz
}

Volume 31, numéro 1, printemps 2003

Difficultés de communication entre parents et intervenants :

éléments d'une sémiotique interdiscursive

URI : https://id.erudit.org/iderudit/1079515ar

DOI : https://doi.org/10.7202/1079515ar

Aller au sommaire du numéro

Éditeur(s)

Association canadienne d'éducation de langue française

ISSN

0849-1089 (imprimé)

1916-8659 (numérique)

Découvrir la revue

Citer cet article

Chatelanat, G., Panchaud Mingrone, I. \& Niggl Domenjoz, G. (2003). Partenariat entre professionnels et parents d'enfants avec une déficience intellectuelle : expériences et attentes des parents. Éducation et francophonie, 31(1), 40-55. https://doi.org/10.7202/1079515ar
Résumé de l'article

Afin de contribuer à préciser la notion de partenariat si largement évoquée aujourd'hui pour désigner les rapports entre les parents d'enfants handicapés et les professionnels, la recherche présentée, dans cet article, étudie le point de vue des parents, tente d'inventorier leurs expériences et leurs souhaits, identifie les difficultés qu'ils rencontrent et les moyens trouvés pour dépasser ou atténuer ces difficultés. Étant donné que cette recherche est en cours, nous ne présenterons comme résultats que ceux de la phase exploratoire, c'est-à-dire l'analyse des témoignages écrits et publiés par les parents eux-mêmes.
Tous droits réservés @ Association canadienne d'éducation de langue française, Ce document est protégé par la loi sur le droit d’auteur. L’utilisation des 2003 services d'Érudit (y compris la reproduction) est assujettie à sa politique d'utilisation que vous pouvez consulter en ligne.

https://apropos.erudit.org/fr/usagers/politique-dutilisation/ 


\title{
Partenariat entre professionnels et parents d'enfants avec une déficience intellectuelle : expériences et attentes des parents
}

\author{
Gisela CHATELANAT \\ Université de Genève, Genève (Suisse) \\ Isaline PANCHAUD MINGRONE \\ École d'études sociales et pédagogiques, Lausanne (Suisse) \\ Gertrude NIGGL DOMENJOZ \\ Université de Genève, Genève (Suisse)
}

\section{RÉSUMÉ}

Afin de contribuer à préciser la notion de partenariat si largement évoquée aujourd'hui pour désigner les rapports entre les parents d'enfants handicapés et les professionnels, la recherche présentée, dans cet article, étudie le point de vue des parents, tente d'inventorier leurs expériences et leurs souhaits, identifie les difficultés qu'ils rencontrent et les moyens trouvés pour dépasser ou atténuer ces difficultés. Étant donné que cette recherche est en cours, nous ne présenterons comme résultats que ceux de la phase exploratoire, c'est-à-dire l'analyse des témoignages écrits et publiés par les parents eux-mêmes. 


\section{ABSTRACT}

\section{Partnership between professionals and parents of mentally retarded children : parents' experiences and needs}

In order to contribute to clarifying the idea of partnership so widely used today to describe the relationships between parents of handicapped children and professionals, the research presented in this article studies the parents' point of view, in an attempt to make an inventory of their experiences and their wishes, identify the difficulties they encounter and the means found to overcome or alleviate these difficulties. Since this research is still in progress, we will present the results of this exploratory phase, analyzing testimonials that are written and published by the parents themselves.

\section{RESUMEN}

\section{Cooperación entre los profesionistas y los padres de familia con niños que presentan una deficiencia intelectual : experiencias y esperanzas de los padres de familia}

Con el fin de contribuir a la precisión de la noción de cooperación que actualmente se evoca con tanta frecuencia para designar las relaciones entre los profesionistas y los padres de familia que tienen niños minusválidos, presentamos en este artículo una investigación que aborda el punto de vista de los padres de familia, tratando de inventariar sus experiencias y sus esperanzas, identificando las dificultades que confrontan y los medios utilizados para rebasar o atenuar dichas dificultades. Dado que esta investigación aun está en curso, sólo presentamos los resultados obtenidos durante la fase exploratoria, es decir, el análisis de los testimonios escritos y publicados por los padres de familia.

\section{Introduction}

Au cours de deux dernières décennies, il est devenu banal d'évoquer l'importance de l'apport des parents d'enfants vivant avec une déficience aux activités thérapeutiques et éducatives proposées par des professionnels. Déjà dans les années soixante-dix, les premières évaluations des programmes de pédagogie compensatoire (Bronfenbrenner (1974)), mis en place aux États-Unis au cours de la décennie précédente, démontraient la supériorité des programmes impliquant activement la 
famille. Depuis cette période, les recherches sur la famille se sont multipliées et développées dans de nombreuses directions, modifiant graduellement la place faite aux parents dans la collaboration avec les professionnels.

Le modèle écosystémique proposé par Bronfenbrenner (1977); (1979); Gallimore et alii (1989); Lambert \& Lambert-Boite (1993) a été parmi les plus importants outils permettant de réorganiser les recherches, les réflexions sur l'enfant handicapé et sa famille, l'organisation de sa prise en charge, etc. Cette perspective théorique met en évidence les influences qu'exercent différents sous-systèmes sociaux sur l'enfant. La famille est l'un des microsystèmes dans lesquels évolue ce dernier. L'enfant et la famille agissent et réagissent toujours en fonction d'autres sous-systèmes plus éloignés, mais déterminants.

En appliquant ce cadre d'analyse à l'éducation spéciale, il est important de préciser que ni la nature de la déficience, ni les démarches pour réduire des incapacités ne peuvent être considérées indépendamment de l'écosystème familial. Le bien-être de l'enfant et ses capacités d'évolution sont liés à l'évolution de la famille dans son ensemble. Les ressources professionnelles doivent s'allier aux ressources familiales de façon à déboucher sur un projet cohérent.

Dès lors, il s'agit de comprendre la situation particulière de chaque famille, lui faire confiance, lui restituer responsabilités et compétences. Dunst et ses collaborateurs (Dunst \& Trivette (1988); Dunst, Trivette \& Deal (1994)) ont proposé les termes " empowering" et « enabling " pour abonder dans le sens de cette mobilisation des ressources familiales. Le terme "Enabling", traduit par Bouchard et alii (1994) par "le principe de l'autodétermination ", se réfère à la capacité d'acquérir des compétences pour assumer des responsabilités ou des décisions, et affirmer son rôle de parent en soutenant ses propres objectifs et ses attentes à l'égard des "prestataires de services »; tandis que le terme " empowering », traduit par « le principe d'appropriation ", renvoie plutôt à l'acquisition de la confiance en ses propres ressources pour réussir dans cette entreprise.

L'apparition des projets éducatifs individualisés (PEI) et des plans de services (PSI) dans les pratiques des services spécialisés (Boisvert (1990); McGonigel et alii (1991)) offre aujourd'hui un moyen pour réaliser cette association indispensable des ressources respectives des parents et professionnels (Chatelanat \& Panchaud Mingrone (1996)). Faire participer les parents à cette démarche d'élaboration du projet éducatif sert à la fois à la concrétisation des principes d'appropriation et d'autodétermination, et donne l'occasion d'apprendre à travailler ensemble, à clarifier les attentes et les rôles de chacun ainsi qu'à négocier ouvertement et respectueusement les désaccords inévitables (Singer \& Powers (1993)).

Mais les pratiques éducatives, dans notre pays du moins, ne semblent pas évoluer en synchronie avec les développements de la recherche. Pour ceux qui sont sur le terrain depuis de nombreuses années, en tant que chercheur ou praticien, force est de constater que si le langage a changé, les relations entre parents et professionnels restent dans bien des situations, pour les uns comme pour les autres, difficiles, conflictuelles et surtout peu articulées. Les modalités de participation offertes aux parents dans le cadre des programmes éducatifs et thérapeutiques sont souvent 
encore fixées unilatéralement par les institutions et elles n'offrent aux parents qu'une mince marge de négociation.

Pourtant, il devient urgent de faire évoluer ces pratiques traditionnelles, non seulement pour réduire l'écart entre ces dernières et les recommandations issues des recherches, mais aussi pour éviter l'aggravation de ces tensions entre professionnels et parents. Le contexte social a changé; les parents d'enfants handicapés ont pris conscience de leur droit; beaucoup d'entre eux ne veulent plus de l'expert tout puissant et de l'institution éducative qui se charge de tout sans pratiquer adéquatement la négociation avec les usagers. L'amorce d'un processus d'intégration des enfants handicapés dans les lieux d'éducation ordinaires modifie la position des parents; ceux-ci n'ont plus pour seuls interlocuteurs les institutions spécialisées; ils prennent conscience du rôle actif qu'ils ont à jouer dans les décisions éducatives concernant leurs enfants (Turnbull \& Turnbull (1990); Bouchard, Boudreault, Pelchat et LalondeGratton (1994)). C'est avec une telle réflexion qu'émerge de façon insistante le sens du terme de partenariat.

«Le partenariat est réalisé quand la famille et les professionnels se respectent, se font confiance et communiquent ouvertement les uns avec les autres " (Turnbull (1990, p. 144)). Cette définition a l'avantage de susciter des regards particuliers sur des significations attribuées tant par les parents que par les professionnels à des termes comme confiance, respect, ou communication ouverte. Dans un premier temps, il paraît important de se libérer des déclarations d'intention, pour aller en profondeur, à la recherche de modèles susceptibles de caractériser les interactions entre parents et professionnels et de faire apparaître les illustrations de ces interactions, les récits faits par les parents, ou l'expression de leur subjectivité. Dans cette perspective, nous avons lancé une recherche qui est actuellement en cours et dont nous allons brièvement esquisser le déroulement. Nous présenterons ensuite plus en détails la phase exploratoire, la seule achevée jusqu'à ce jour, en nous arrêtant sur les principaux résultats obtenus.

En donnant la parole aux parents, le but général de l'étude est, d'une part, de décrire les expériences et les attentes des parents par rapport à la collaboration avec les professionnels impliqués dans la prise en charge de l'enfant vivant avec une déficience intellectuelle, et, d'autre part, de développer une réflexion quant aux moyens qui pourraient faciliter l'instauration plus généralisée de rapports entre partenaires mieux adaptés aux besoins réels de chaque famille.

Cette recherche se déroule en quatre étapes distinctes. Nous avons choisi une procédure « en cascade", qui devrait nous permettre à chaque étape de cibler plus précisément les thèmes prédominants qui émergent des récits du vécu et des perceptions parentales. Ainsi, les résultats les plus prégnants d'une étape de la recherche seront repris et étudiés d'une manière plus approfondie dans l'étape suivante. L'analyse de chaque étape devrait tenir compte de toute la richesse et de la diversité des récits des parents, mais une réduction des données sera effectuée pour la suite.

Dans la phase exploratoire, le but principal est de mettre en évidence différents aspects des relations entre professionnels et parents, et d'inventorier les préoccupa- 
tions de ces derniers telles qu'elles ressortent de documents écrits par eux-mêmes, c'est-à-dire des témoignages publiés dans des livres et les bulletins d'associations de parents. Une analyse de contenu des passages pertinents dans ces documents a permis de dégager une série de questions, de thèmes et de préoccupations. Nous présentons plus loin quelques résultats de cette phase exploratoire.

Dans la deuxième étape, le but principal est de mieux connaître et comprendre, dans une région de Suisse romande (le canton de Vaud), les perceptions que les parents d'enfants handicapés ont aujourd'hui des différents professionnels impliqués dans la prise en charge de leur enfant. Il s'agit de les entendre au sujet de leurs relations avec ces professionnels, de leurs expériences concrètes et leurs souhaits à l'égard de ces échanges. Il nous importe notamment de connaître les perspectives parentales quant aux facteurs qui facilitent à leur avis ces relations, sur les difficultés qu'ils ont rencontrées et les explications qu'ils en donnent. Il s'agit également d'inventorier les stratégies qu'ils ont tenté d'élaborer pour développer des relations de collaboration satisfaisantes et pour en surmonter les obstacles.

Pour cette étape, trois modes successifs de récolte des données sont prévus :

a. des questionnaires,

b. des entretiens individuels,

c. et des entretiens de groupes.

Ces différentes méthodes suivent également notre procédure " en cascade ", car elles nous permettent, chaque fois, de cibler progressivement les éléments saillants mis en évidence par la méthode déjà utilisée.

La dernière étape devrait plutôt avoir une fonction d'ouverture vers des investigations complémentaires à la nôtre. L'ambition est de confronter le discours des professionnels à quelques-unes des données réunies dans les trois premières étapes, afin de mesurer l'écart entre les perceptions de ces deux catégories d'acteurs. Nous devrions nous adresser surtout à des groupes de professionnels pour pouvoir amorcer un dialogue - du moins virtuel - entre partenaires.

Comme nous l'avons mentionné plus haut, nous présentons et discutons cidessous les principaux résultats de cette phase exploratoire.

\section{Témoignages écrits de parents}

L'inventaire des expériences relatives aux contacts et interactions avec les différents professionnels impliqués dans la prise en charge de l'enfant handicapé telles que les parents les ont vécues et décrites eux-mêmes, devrait donc contribuer à nous orienter dans la préparation des entretiens prévus dans les phases ultérieures d'une recherche plus large. Dans cette perspective, nous avons analysé d'une part, des livres écrits par des parents, et, d'autre part, des articles publiés dans des bulletins d'associations de parents en Suisse romande. 


\section{Le point de vue des parents au travers de livres-témoignages}

L'analyse de livres a l'avantage de replacer le rapport entre parents et professionnels dans le contexte plus large de la vie et de l'évolution d'une famille particulière. Les récits parentaux introduisent une dimension temporelle, car ils s'étendent généralement sur plusieurs années et les collaborations avec les professionnels se situent dans le cadre plus étendu des pratiques et préoccupations familiales. Chacun des témoignages est la trace subjective d'une tranche de vie qui peut nous faire partager non seulement des événements ponctuels, mais surtout des processus contextualisés, comme par exemple le processus de diagnostic ou celui de l'ajustement familial. Dans ces deux exemples, le rôle que les professionnels tiennent aux yeux des parents est certainement primordial. (Martin et alii (1993); Bouchard, Boudreault, Pelchat \& Lalonde-Graton (1994); Corbet \& Greco (1994)).

Nous avons procédé de la manière suivante : à partir d'une trentaine de livres étudiés, nous avons effectué une sélection pour ne retenir que neuf récits (voir références bibliographiques). En effet, nous avons éliminé les récits trop anciens (enfants nés avant 1969) pour éviter des contextes historiques trop différents de celui vécu par les parents qui participeront aux phases suivantes de la recherche. Puisque cette dernière concerne exclusivement des parents dont l'enfant est concerné par une déficience intellectuelle ou un polyhandicap, nous avons exclu des livres à propos d'enfants vivant avec des déficiences sensorielles ou motrices. Il va de soi que les récits doivent contenir des passages consacrés aux rencontres entre parents et professionnels. Nous avons donc identifié ces passages et nous les avons analysés à l'aide de deux types de fiches : des fiches d'identification et des fiches analytiques. Les premières résument brièvement le témoignage et décrivent l'auteur, l'enfant, l'époque, le pays; les deuxièmes retiennent, pour chaque extrait concernant une rencontre entre les parents et des professionnels, les éléments suivants :

\begin{tabular}{|c|c|}
\hline Acteurs & $\begin{array}{l}\text { Qui sont les acteurs impliqués dans la rencontre avec le ou } \\
\text { les professionnels? }\end{array}$ \\
\hline Lieu de rencontre & Où l'action se déroule-t-elle? \\
\hline Motifs de la rencontre & Pourquoi les acteurs se rencontrent-ils? \\
\hline Âge de l'enfant/année & Quand l'action se déroule-t-elle? \\
\hline Sujet/thème & Quel est le sujet? Sur quoi porte l'interaction? \\
\hline $\begin{array}{l}\text { Type d'interaction : } \\
\text { directe ou indirecte }\end{array}$ & $\begin{array}{l}\text { L'extrait porte-t-il sur une communication directe avec un professionnel } \\
\text { directe ou indirecte ou s'agit-il d'un commentaire ou d'un jugement } \\
\text { sur ces derniers? }\end{array}$ \\
\hline $\begin{array}{l}\text { Jugement positif, } \\
\text { négatif, neutre ou } \\
\text { indéfini }\end{array}$ & Comment le parent se représente-t-il la rencontre? \\
\hline Conflit & $\begin{array}{l}\text { S'agit-il d'une situation insatisfaisante ou d'une situation satisfaisante } \\
\text { aux yeux des parents? }\end{array}$ \\
\hline $\begin{array}{l}\text { Résolution-fin bien } \\
\text { résolue, mal résolue, } \\
\text { non résolue ou indéfinie }\end{array}$ & Comment le conflit est-il résolu? \\
\hline
\end{tabular}




\section{Résultats et discussion}

L'analyse des fiches d'identification nous permet de décrire quelques éléments clefs issus de neuf situations retenues. Tous les récits, sauf un, commencent avec la naissance de l'enfant, et trois récits se prolongent jusqu'à l'adolescence. Tous se situent dans des pays industrialisés, occidentaux : cinq récits se déroulent en France, et un récit respectivement en Suisse, en Allemagne, en Italie et aux États-Unis.

En ce qui concerne les enfants, il s'agit de cinq garçons et de trois filles, dont quatre enfants avec une trisomie 21 , un enfant avec des traits autistiques, un enfant avec une maladie dégénérative, un enfant IMC, microcéphale, polyhandicapé et un enfant handicapé mental et physique. Un des enfants est enfant unique; il a été adopté à l'âge de 4 ans. En ce qui concerne les autres familles, le nombre d'enfants par famille varie entre deux et quatre. Une majorité des parents exerce une profession exigeant des études supérieures. Cinq mères exercent leur profession au moins à temps partiel et quatre mères sont mères au foyer tout au long du récit.

Au total, 112 épisodes (ou rencontres) entre parents et professionnels sont inventoriés et classés selon les critères mentionnés plus hauts.

\section{Professionnels de la santé versus professionnels de l'éducation}

Frappées par le grand nombre et la variété des professionnels (personnes différentes, professions différentes) que les parents rencontrent au sujet de leur enfant et pour mieux comprendre les tendances qui se dégagent, nous avons organisé les fiches en séparant les professionnels mentionnés par les parents en deux groupes :

1. Les professionnels de la santé : sont considérés comme professionnels de la santé les médecins, infirmières, sages-femmes, kinésithérapeutes, physiothérapeutes et les psychologues rattachés à un service hospitalier.

2. Les professionnels de l'éducation : sont considérés comme professionnels de l'éducation les pédagogues des services d'intervention précoce, les éducateurs de la petite enfance, les enseignants et les éducateurs spécialisés, instituteurs, professeurs, maîtres d'appui, les directeurs d'écoles et les psychologues rattachés à une école.

En regroupant les situations de malaise, les situations satisfaisantes, ainsi que les situations neutres ou indéfinies pour chaque groupe de professionnels, les analyses aboutissent à deux constats principaux :

- Premièrement, les parents mentionnent beaucoup plus fréquemment des rencontres avec les professionnels de la santé (85 rencontres mentionnées) que celles avec les professionnels de l'éducation (27 rencontres mentionnées);

- Deuxièmement, les rencontres avec les professionnels de la santé sont souvent décrites négativement, c'est-à-dire $51 \%$ (44 rencontres) d'entre elles sont perçues négativement; par contre, rares sont les critiques adressées aux professionnels de l'éducation, c'est-à-dire deux rencontres seulement sont vécues comme des situations de conflit ou de malaise. 
Une première hypothèse serait que les contacts entre ces derniers et les parents se déroulent souvent pendant une période de crise, telle que l'annonce de la déficience de l'enfant. En effet, parmi les témoignages, nous trouvons une seule situation où les parents tiennent en estime le pédiatre qui a annoncé la déficience de leur enfant. Cependant, même cette situation satisfaisante est précédée d'une situation de malaise à l'égard du gynécologue et suivie d'une autre, jugée négative, concernant la rencontre avec un généticien.

Une deuxième hypothèse porte à penser que le nombre élevé de jugements négatifs sur les rencontres avec les professionnels de la santé est dû en partie au fait que ces contacts sont très souvent ponctuels, limités à une ou deux rencontres, ce qui est insuffisant pour établir une relation de confiance et de compréhension (Beckman (1996)). Après vérification, en ne retenant que les rencontres directes entre parents et professionnels de la santé, il s'est en effet avéré que 25 rencontres sur 31 ont été des rencontres initiales.

Tout en admettant qu'il est difficile pour un professionnel d'établir des interactions satisfaisantes lors de rencontres ponctuelles et de surcroît dans une situation de crise, il est préoccupant que les professionnels de la santé semblent accuser de si importantes lacunes sur le plan de la préparation individuelle (Corbet \& Greco (1994)).

Les jugements plus positifs sur les professionnels de l'éducation dans le contexte d'interactions suivies font donc supposer qu'une certaine continuité et une plus longue durée des relations, permettent de construire progressivement une collaboration qui puisse alors satisfaire les parents grâce à des ajustements réciproques. Par ailleurs, les rencontres sont moins souvent situées dans des périodes de crise.

\section{Le " bon " et le " mauvais " professionnel}

À partir d'une nouvelle analyse des passages inventoriés sur les fiches, nous avons élaboré une sorte de "portait-robot » des «bons " et « mauvais " professionnels décrits par les parents, tout en gardant la séparation entre professionnels de la santé et professionnels de l'éducation.

En définitive, les qualités relationnelles, dans un sens large, sont vraiment de loin les qualités que les parents souhaitent trouver chez un professionnel de la santé.

\begin{tabular}{|c|c|}
\hline $\begin{array}{l}\text { Éléments descriptifs d'un professionnel } \\
\text { de la santé qui ne satisfont pas } \\
\text { aux attentes des parents }\end{array}$ & $\begin{array}{l}\text { Éléments descriptifs d'un professionnel } \\
\text { de la santé qui satisfont aux attentes } \\
\text { des parents }\end{array}$ \\
\hline $\begin{array}{l}\text { - } \quad \text { froid, arrogant, dur, égoïste } \\
\text { - } \quad \text { insensible, manque de tact } \\
\text { - } \quad \text { ne prend pas le temps } \\
\text { - } \quad \text { ne s'intéresse pas à l'enfant, à sa } \\
\text { - } \quad \text { nouffrance } \\
\text { ne se concentre que sur les aspects } \\
\text { négatifs } \\
\text { manque de respect (de la vie privée, } \\
\text { des besoins, compétences et contraintes } \\
\text { des parents). }\end{array}$ & $\begin{array}{l}\text { - } \quad \text { est humain, chaleureux, aimable } \\
\text { - a une voix basse et calme } \\
\text { - } \quad \text { a un regard doux et lumineux } \\
\text { - } \quad \text { an juste } \\
\text { - } \quad \text { aime les enfants, s'intéresse à eux } \\
\text { - } \quad \text { respecte leurs compétences et faiblesses } \\
\text { - } \quad \text { sait encourager, réconforter. }\end{array}$ \\
\hline
\end{tabular}


Au moins sept passages accusent le professionnel de la santé d'une attitude froide ou arrogante; huit mentions sont faites d'une attitude d'indifférence voire de désintérêt à l'égard de l'enfant. Pratiquement tous les parents déplorent le manque de respect face à leur vie privée, à l'égard de leurs besoins de parents ou à l'égard de leurs compétences parentales.

«Les mains croisées sur son bureau, il m'examina froidement un court instant; j'espérais un peu d'humanité, mais les yeux au regard implacable se posèrent sur Sophie avec une évidente indifférence et ses paroles tombèrent comme la lame d'un couperet. » (Jolly (1980, p. 61))

Le " mauvais " professionnel a une attitude d'indifférence à l'égard de l'enfant ou à l'égard de sa souffrance. Le «bon professionnel » montre de l'intérêt et de l'affection pour l'enfant. L'expression "être chaleureux» est mentionnée presque par tous les parents.

"C'est un beau garçon dit la neuropsychiatre qui le suit aujourd'hui. «Il donne une sensation de vie" et son regard, alors qu'elle prononce ces mots, devient incroyablement doux et lumineux. Je lui suis très reconnaissante pour ces paroles. Mais ce qui me touche le plus, c'est qu'elle est capable de le suivre, non pas comme un " cas ", mais comme un être humain auquel elle voue, sans aucun doute, une grande affection. Son attitude est rare. Elle est surtout bien éloignée de celle de nombreux autres médecins que j'ai rencontrés auparavant. » (Basano (1989))

En outre, un certain nombre de passages des récits reflète le besoin des parents d'obtenir du professionnel de la santé des explications compréhensibles («savoir expliquer $»$.

"Quand on est amené à faire basculer la vie des gens, on devrait pouvoir mesurer l'impact possible de ses paroles, surtout lorsqu'on s'adresse à des parents dont on ne connaît absolument rien et surtout pas la résistance psychologique. Ce pédiatre-là choisit de me parler d'abord le langage scientifique. Sans doute était-il plus à l'aise ainsi pour surmonter son propre malaise? Ce langage est pourtant bien abrupt, d'autant que pour moi qui suis familière du grec ancien, les termes médicaux ne sont pas, en général, trop mystérieux. Cela évidemment il ne pouvait pas le savoir! »(Debarbieux (1990, pp .19-20)).

Et les parents s'attendent également à être informés, écoutés et considérés : "C'était un peu comme si j'étais la responsable de tes maux, celle qui ne te fait que du mal, comme si ma présence allait te perturber. [...] Et il en a toujours été ainsi, elle me donnait au compte-gouttes quelques informations malgré elle, mais refusait totalement la moindre collaboration. " (FirinoMartell (1995, pp. 35-36)) 
"Ici il n'y a pas de grand patron suivi de médecins complaisants, qui ouvrent le parapluie et pour rien au monde ne contrediraient leur patron. Ici, les décisions sont prises au grand jour, les parents sont considérés comme des adultes et y sont associés. » (Firino-Martell (1995, p. 40))

Si les compétences professionnelles liées à la technique du métier sont également mentionnées, elles sont souvent accompagnées de remarques indiquant que ces compétences techniques sont indispensables, certes, mais qu'elles doivent nécessairement être doublées de qualités humaines.

« En plus de sa compétence médicale, je sais que je peux faire appel à ses qualités humaines. C'est de ça dont j'ai besoin, pas seulement d'un excellent technicien. » (Lafon (1989, p. 28).

En ce qui concerne le «bon " professionnel de l'éducation, la qualité mentionnée le plus souvent est " être disponible ». À propos des qualités relationnelles, nous constatons que les parents expriment comme conviction qu'il est important de savoir établir un bon contact avec un élève difficile et de ne pas le considérer seulement comme un " cas ", mais avant tout comme un enfant. La troisième personne, l'enfant, prend beaucoup d'importance. Plusieurs parents mentionnent le besoin de pouvoir parler régulièrement avec l'enseignant au sujet de leur enfant.

"Les enseignantes étaient jeunes et pleines d'enthousiasme. Elles manquaient peut-être de l'expérience, mais elles avaient une forte envie d'apprendre et une grande, très grande disponibilité. » (Basano (1989, p. 69))

« Parfois quand j'allais le chercher, je trouvais Rosie découragée et fatiguée. Mais elle ne me demanda jamais de le garder à la maison. Nous en parlions ensemble, en cherchant des solutions et des réponses aux problèmes. « Elle était là » et Nicola la sentait proche de lui. Elle l'obligeait à rester au milieu des autres et elle exigeait de lui tout ce qu'il était en mesure de donner. De cette façon, il retrouvait confiance en lui, parce qu'elle avait confiance en lui.» (Basano (1989, p. 90))

Des tendances riches et hétérogènes se dégagent de ces récits. Nous constatons en effet que mention est faite des professionnels de la santé beaucoup plus souvent et d'une manière plus variée. Nous pensons que le processus de révélation de la déficience y est pour beaucoup. L'accouchement et la période de diagnostic, de soins, de contacts avec les médecins, infirmières et puéricultrices créent un cumul de rencontres qui restent gravées dans le souvenir des parents. Si la déficience n'est révélée que progressivement, cette période est vécue comme angoissante et frustrante. Il n'est pas étonnant que les parents expriment leur besoin d'information dans un contexte d'empathie et de chaleur humaine. Il est d'autant plus préoccupant que la préparation personnelle des médecins, psychologues et infirmières reste insuffisante pour permettre au personnel médical et paramédical de faire face à cette situation (Corbet \& Greco (1994); Martin et alii (1993)). C'est avec les professionnels de 
l'éducation, plus qu'avec les médecins que les parents semblent établir des relations de collaboration égalitaires. Lorsque l'enfant évolue dans une collectivité, sans leur présence, plusieurs parents réalisent la nécessité des échanges réguliers et des rapports de coordination, de confiance et de respect mutuel.

D'une manière générale, c'est la force, la clairvoyance et la détermination qui permettent aux familles de traverser des périodes difficiles et souvent dramatiques pour non seulement retrouver un équilibre, mais aussi se considérer enrichies par les épreuves (sans fin), de même que par la vie en compagnie de leur enfant. Cette image reflétée par les parents contraste avec les représentations négatives qui souvent habitent les professionnels. Pour ces derniers, les interactions avec les parents d'un enfant avec une déficience se construisent en principe sur un arrière-plan de deuil, de non acceptation de la déficience, de souffrance. Déjà là s'étalent des présupposés qui empêchent ces professionnels de rencontrer les parents sur une base d'égalité. Une telle attitude peut être source de difficulté. Aussi semble-t-il justifié de se demander si la souffrance des parents, perçue par les professionnels, n'est pas entretenue par des difficultés extérieures, bien plus souvent que par des facteurs intrapsychiques touchant ces parents.

\section{Le point de vue des parents au travers d'articles parus dans les bulletins d'associations}

Dans un deuxième temps, nous avons pris connaissance d'articles parus ces dix dernières années dans les revues d'associations de parents de personnes mentalement handicapées ou IMC en Suisse romande, en y cherchant des publications, ou parties d'articles, traitant des relations entre parents et professionnels. Cette démarche est partie de l'idée selon laquelle les associations de parents demeurent des lieux privilégiés où les parents peuvent trouver un appui. Ils pourraient trouver de plusieurs façons un appui : réflexion entre parents qui vivent des situations similaires, échanges d'informations, connaissance de leurs droits, cours de formation pour parents... Les associations joueraient alors un rôle important dans ce que Dunst et ses collaborateurs (Dunst et alii (1986), (1988)) ont appelé des processus d'appropriation ("empowering ») et d'autodétermination (" enabling»). Selon ces auteurs, c'est au travers de ces deux processus que les parents mobilisent leurs ressources, développant les compétences et l'assurance nécessaire, afin de devenir plus autonomes dans la prise de responsabilités et de décisions, pour pouvoir défendre leurs propres buts en regard des prestataires de services.

Bien entendu, les bulletins des associations s'adressent surtout aux membres et parents dans le but de les rassembler et surtout de les informer sur différents aspects importants concernant leurs droits, ainsi que les possibilités de formation et de loisirs pour leur enfant handicapé. En outre, ils concrétisent le rôle d'écoute et de soutien d'une association. C'est dans ce sens que les bulletins peuvent être un médium qui permet aux parents de s'exprimer à propos de leurs joies, leurs besoins et leurs problèmes, liés à la vie de cet enfant, adolescent ou adulte, vivant avec une déficience 
intellectuelle, mais aussi de partager des préoccupations par rapport à certains sujets qui leur tiennent particulièrement à cœur. La relation que les parents entretiennent avec des professionnels impliqués dans la prise en charge complète ces sujets. Tous les passages pertinents inventoriés (dont ceux faisant référence aux rapports entre parents et professionnels), répondent en définitive à deux grandes tendances : celle des expériences négatives (critiques et reproches) et celle des expériences satisfaisantes.

\section{À propos des constats}

Nous avons eu, en définitive, la surprise de constater que notre thème occupe très peu de place dans ces bulletins. En effet, nous n'avons trouvé que deux numéros (Dialogue parents-professionnels et Pour un dialogue authentique). En ce qui concerne les paragraphes consacrés à notre thématique, nous en avons trouvé un petit nombre. C'est surtout dans le cadre de grands thèmes (l'intégration scolaire, placement en internat, l'autisme) qu'il est fait allusion aux relations parents-professionnels, mais d'une manière très succincte. Il arrive aussi que certains rapports annuels d'activités des associations, de comptes rendus de discussions en commission ou de rapports concernant une participation à une formation ou à un congrès consacrent quelques lignes aux difficultés dans la relation parents-professionnels. Mais peu de traces du contenu de ces discussions subsistent.

Dans l'ensemble des six bulletins et sur une dizaine d'années, nous n'avons pu trouver que vingt-quatre passages qui font allusion à notre sujet. En nous demandant pourquoi les bulletins d'associations de parents ne consacrent pas plus de place au thème du partenariat, nous identifions plusieurs hypothèses. Premièrement, un bulletin d'association se donne probablement la mission d'encourager ses membres; cela peut expliquer la grande retenue quant à la description des situations insatisfaisantes et aux critiques ouvertes en général. Par ailleurs, on peut penser que beaucoup de membres d'une association s'exposent en prenant la plume et craignent d'indisposer des interlocuteurs professionnels avec lesquels ils entretiennent encore des relations. Beaucoup de parents pratiquent sans doute une certaine autocensure. Nous avons d'ailleurs constaté que les parents ont plus d'aisance à formuler leurs expériences négatives lors des réunions de groupes et lorsqu'ils peuvent choisir un porte-parole. Dans la majorité des passages extraits des bulletins, c'est-à-dire dixneuf, les parents s'expriment d'une manière négative ou critique, par rapport aux rencontres ou relations de collaboration avec les professionnels. Cette fois, professionnels de la santé et professionnels de l'éducation sont mentionnés à proportion presque égale : parmi ces appréciations négatives, neuf concernent les professionnels de la santé et dix les professionnels de l'éducation. En ce qui concerne le contenu ou le motif des rencontres avec les professionnels de la santé, on retrouve, comme pour les livres, la prédominance de situations liées à l'annonce du handicap et au processus de diagnostic.

« [...] je crois, qu'il (le pédiatre à la maternité) voulait me faire comprendre que cet enfant avait très peu de chance de survie, c'est pour cela qu'il noircissait le tableau déjà très noir. Et j'ai pris son attitude pour une entreprise 
de dénigrement et de démolition. [...] Le pauvre (le mari), il avait eu droit à une description tellement horrible que, lorsque l'infirmière est partie chercher le bébé à mon réveil, il est sorti avec elle pour lui demander de ne pas m'apporter le monstre qui lui a été décrit! »

(Mère d'un enfant trisomique atteint d'une cardiopathie, âgé de 15 ans au moment de ce témoignage).

Pour les dix passages qui parlent des professionnels de l'éducation, il s'agit essentiellement d'insatisfactions dans des situations d'intégration scolaire et sociale ou dans celles liées au placement de l'enfant en internat.

« Nous avons connu dans l'enseignement spécialisé un échange fantastique entre les parents et l'école. Dès l'école primaire (ordinaire), les parents sont exclus de l'école. Pour des enfants handicapés, le lien entre l'école et la famille est très important. "

(Mère d'un garçon avec un retard psychomoteur, âgé de neuf ans au moment du témoignage).

\section{Conclusion}

Cette étude met tout d'abord en évidence la pauvreté du matériel dans les "livres-témoignages", mais surtout dans les journaux d'associations. Ce résultat confirme la nécessité de rendre le point de vue des parents plus visible. Les parents doivent être l'une des ressources essentielles pour apporter un éclairage sur ce que peut être le partenariat entre parents et professionnels.

Un deuxième enseignement que nous tirons de cette étude exploratoire est que le thème du partenariat est rarement abordé en général, mais qu'il est étroitement lié, sinon subordonné, aux situations dans lesquelles il doit se réaliser: annonce de la déficience, transition d'une structure à l'autre, intégration scolaire et sociale, situations d'apprentissage ou de thérapie... Il est probable que, à part quelques principes de base, les modalités de collaboration attendues et souhaitées par les parents soient différentes selon les situations qui se présentent et les problèmes qui sont à résoudre. Pour les entretiens, nous retenons les questions concernant le partenariat abordées, dans ce contexte, non seulement comme des caractéristiques et besoins de familles particulières, mais aussi comme des repères par rapport à ce que la famille perçoit comme besoins prioritaires de son enfant à un moment donné de son développement et par rapport à des situations concrètes. De même, les attentes et souhaits des parents ne sont pas identiques pour tous les partenaires. Ils sont au contraire spécifiques à des fonctions et des rôles professionnels.

Il est important de retenir en définitive que la difficulté de trouver les moyens pour que les parents s'expriment sur le thème de la collaboration, notamment la collaboration insatisfaisante, demeure un objet de préoccupation majeure, alors même que toutes sortes de contingences (absence de choix, pauvreté de la législation, dévalorisation de leur situation présente) limitent leur évaluation de la situation. 
L'exploration de documents écrits par les parents montre bien que ces derniers ne s'autorisent que rarement, en tant qu'individus comme en tant que membres d'une association, à formuler des revendications ou même des souhaits de changements.

\section{Références bibliographiques}

\section{Références}

Beckman, P.J. (Ed.) (1996). Strategies for working with families of young children with disabilities. Baltimore, Maryland : Paul H. Brookes.

Bouchard, J.-M., Boudreault, P., Pelchat, D., Lalonde-Graton, M. (1994). Déficiences, incapacités et handicaps : Processus d'adaptation et qualité de vie de la famille. Montréal : Guérin.

Bronfenbrenner, U. (1979). The ecology of human development. Cambridge : Harvard University Press.

Chatelanat, G., Panchaud Mingrone, I. (1996). Partenariat entre les parents d'enfants handicapés et les professionnels : expériences et attentes des parents. In Petites personnes, grands projets : Projets pédagogiques pour jeunes enfants handicapés. Chatelanat, G. et Panchaud Mingrone, I. (Eds.), Lucerne : SZH/SPC, 1996.

Corbet, E., Greco, J. (1994). Annonce du handicap : Représentations et réalités. Lyon : CREAI Rhone-Alpes.

Dunst, C.J., Trivette, C.M. (1988). Enabling and empowering families : Principles and Guidelines for Practice. Cambridge, MA : Brookline Books.

Dunst, C.J., Trivette, C.M., Deal, A.D. (1994). Supporting \& Strengthening Families. Volume 1 : Methods, Strategies and Practices. Cambridge, MA : Brookline Books.

Gallimore et alii (1989). The social construction of ecocultural niches : Family accomodation of developmentally delayed children. In American Journal of Mental Retardation, Vol. 3 (94), pp. 216-230.

McGonigel, M.J., Kaufmann, R.K., Johnson, B.H. (1991). Guidelines and recommended practices for the individualized family service plan. Bethesda : NEC*TAS and ACCH.

Lambert, J.-L., Lambert-Boite, F. (1993). Éducation familiale et handicap mental. Fribourg $(\mathrm{CH})$ : Éditions Universitaires. 
Martin, P., Papier. C., Meyer, J. (1993). Le handicap en question : des familles face à la découverte du handicap et l'accompagnement du jeune enfant à domicile. Paris : CTNERHI (flash informations no hors série).

Singer, H.S., Powers, L.E. (eds.) (1993). Families, disabilities and empowerment. Active coping skills and strategies for family interventions. Baltimore : Paul Brookes

Turnbull, A. P., Rutherford Turnbull, H. (1990). Families, professionnals and Exceptionality : a special partnership. Deuxième édition. New York : Merrill, Macmillan Publishing Company.

\section{Livres-témoignages écrits par des parents sélectionnés pour cette étude}

Basano, Julia (1989). Histoire de Nicolas. Meyzieu : Césura Lyon Edition. (Edition originale en italien, 1988)

Debarbieux, Anne-Marie (1990). Son ombre nous éclaire. Accueillir notre enfant trisomique. Paris : Nouvelle Cité.

Delaunet, Gilles (1989). Emmanuel, mystère d'amour, petit prince du royaume. Paris : Office Chrétien des Handicapés (O.C.H.).

Firino-Martell, Thérèse. (1995). Mon enfant citadelle. Paris : Éditions Fayard dans la collection «Les enfants du fleuve».

Jolly, Chantal, (1980). Sophie ma fille ou le combat pour la vie d'un enfant handicapé. Paris : Les Éditions Ouvrières.

Kupfer, Fern (1983). Avant et après Zacharie. Paris : Éditions Robert Laffont. (Édition original, 1982)

Lafon, Monique (1989). Mon enfant, ma douleur, mon bonheur. Paris : Acropole.

Lebeus, Angelika-Martina (1995). Liebe auf den zweiten Blick. Bergisch Gladbach : Gustav Lübbe Verlag GmbH. (Édition originale en allemand, 1989)

Ranc, Jacqueline (1986). Je suis la maman d'un enfant handicapé. Lausanne : Éditions contrastes.

\section{Les bulletins des associations de Suisse romande}

Association genevoise de parents et d'amis de personnes mentalement handicapées. APMH, bulletin de l'Association genevoise de parents et d'amis de personnes mentalement handicapées (trimestriel). Bulletins examinés : mars 1985 - octobre 1997.

Association vaudoise de parents de handicapés mentaux. Coup d'Pouce, bulletin d'information de l'Association vaudoise de parents de handicapés mentaux (trimestriel). Bulletins examinés : juin 1981 - septembre 1997. 
Association valaisanne de parents de personnes handicapées mentales. Main dans la main, bulletin de l'Association valaisanne de parents de personnes handicapées mentales (trimestriel). Bulletins examinés : mars 1985 septembre 1997.

Association neuchâteloise de parents de personnes mentalement handicapées. Bulletin de l'Association neuchâteloise de parents de personnes mentalement handicapées (semestriel). Bulletins examinés : juin 1986 - juin 1997.

Association genevoise en faveur des I.M.C.. Echo, bulletin de l'Association genevoise en faveur des I.M.C. (trimestriel). Bulletins examinés : octobre 1988 septembre 1997.

Ligue vaudoise en faveur des I.M.C.. Contact, bulletin de la Ligue vaudoise en faveur des I.M.C. (trimestriel). Bulletins examinés : mars 1988 - septembre 1997. 